\title{
ANÁLISE DA RENTABILIDADE DE CLIENTES ATRAVÉS DA MARGEM DE CONTRIBUIÇÃO: UM ESTUDO EM UMA EMPRESA DE MÉDIO PORTE DO SETOR MOVELEIRO LOCALIZADA NA SERRA GAÚCHA
}

\author{
C. Hastenteufel ${ }^{1}$; F.Larentis ${ }^{1 *}$ \\ 1 Universidade de Caxias do Sul, 95070-560, Caxias do Sul-RS, Brasil \\ * flarenti@ucs.br
}

Artigo submetido em 01/2015 e aceito em 04/2015

\section{RESUMO}

No atual mercado competitivo, conhecer a rentabilidade de cada cliente é peça chave para o sucesso de uma empresa, mas, para chegar neste percentual, é preciso adotar alguns procedimentos. Em detrimento a isso, o estudo efetuado demonstra os procedimentos a serem seguidos para a determinação e análise da rentabilidade dos principais clientes de uma empresa do setor moveleiro. Nesse contexto o presente trabalho foi realizado pelo método de estudo de caso, com o objetivo de demonstrar a importância do conhecimento da rentabilidade dos clientes com base na margem de contribuição, melhorando dessa forma, de maneira eficiente e eficaz seu processo de gerenciamento. Para tanto será apresentado o referencial teórico sobre a análise da rentabilidade de clientes, os métodos de custeio e mensuração da rentabilidade de clientes através da margem de contribuição. Concluindo é proposta a utilização dos dados obtidos e tal proposta é aplicada na empresa de nome fictício Bella.

PALAVRAS-CHAVE: Margem de Contribuição, Análise de Rentabilidade, Clientes. Métodos de Custeio.

\section{ANALYSING CUSTOMERS PROFITABILITY VIA CONTRIBUTION MARGIN: A STUDY IN A BUSINESS SECTOR OF MEDIUM SIZE FURNITURE LOCATED IN SERRA GAÚCHA.}

\begin{abstract}
In today's competitive market, knowing the profitability of each customer is a key part to the success of a company, but to reach, this percentage, you need to adopt some procedures. This study shows procedures to be followed for the determination and analysis of profitability of major customers of a company in furniture sector. In this context, the present work was carried out by the case study method, to demonstrate the importance of knowing the profitability of customers
\end{abstract}

based on contribution margin, thus improving efficiently and effectively their management process. We will present theoretical framework for the analysis of customer profitability, methods of costing and measurement of existing profitability and the profitability of customers through the contribution margin. As a conclusion, we propose using data and this proposal applied to the Bella fictitious name company.

KEYWORDS: Contribution Margin, Profitability Analysis, Customers, Costing Methods 


\section{INTRODUÇÃO}

Com um mercado global cada vez mais competitivo, as empresas aumentaram sua preocupação em relação ao gerenciamento de seus custos e consequentemente de sua rentabilidade. Nesse contexto torna-se fundamental para a empresa a utilização instrumentos gerenciais que demonstrem de forma adequada, os recursos que estão sendo consumidos por seus clientes, buscando uma diferenciação que possa gerar vantagens competitivas.

Considerando que o cliente também contribui para a geração de riqueza da empresa e não somente o produto, torna-se muito importante uma correta avaliação e gerenciamento de sua rentabilidade, para que dessa forma seja possível adotar novas estratégias na administração e gerenciamento dos negócios.

Tradicionalmente a avaliação de rentabilidade é dada por volume de negociação ou pela margem bruta (SHARMANN, 1996). Entretanto, há uma série de outros custos significativos e que devem ser considerados para avaliar corretamente a rentabilidade de um cliente.

Verificando-se a necessidade de um sistema que apure de maneira correta os custos da empresa para que seja possível avaliar de forma mais precisa e individual cada cliente, permitindo uma classificação por rentabilidade, para que dessa forma a empresa consiga adotar melhores estratégias, ajustando seus níveis de serviço com base nessas diferenças, sugeriu-se então a avaliação através da Margem de Contribuição. Através deste modelo, com base no custeio variável, é alocado aos produtos somente os custos variáveis, os custos fixos são considerados despesas contribuindo diretamente para o resultado.

A partir dessa problemática, o objetivo geral desse trabalho é analisar a rentabilidade de cada cliente através da Margem de Contribuição, em uma empresa de médio porte do setor moveleiro localizada na serra gaúcha permitindo uma melhor gestão de clientes através desses resultados. Como objetivos específicos pretende-se, medir o grau de rentabilidade, conhecer o perfil individual de cada cliente, demonstrar as vantagens de se apurar sua rentabilidade de forma precisa, definir estratégias para a manutenção de clientes rentáveis e, ao mesmo tempo, adequar os custos e níveis de serviços para os clientes menos rentáveis.

Dito isso, acredita-se que mensurar a rentabilidade obtida com os clientes, bem como saber gerenciá-la, pode proporcionar a empresa uma boa oportunidade de rentabilizar suas relações, contribuindo para um aumento na rentabilidade geral, particularmente com as atividades de fabricação de produtos e prestação de serviços, como é o caso da empresa em estudo.

\section{REVISÃO DA LITERATURA}

\subsection{Análise da rentabilidade de clientes}

Na última década o mercado em geral tornou-se muito mais competitivo, com a entrada cada vez maior de produtos de outros países e, associado a isso, o mercado consumidor vem aumentando seu nível de exigência. Diante desse novo cenário, para que as empresas possam continuar competitivas é necessário otimizar seus recursos e ao mesmo tempo oferecer produtos e serviços que atendam de forma adequada a demanda desse novo mercado consumidor.

Diante desses fatos, segundo Guerreiro (1999), os estudos atuais de marketing indicam que as empresas devem dar maior atenção a rentabilidade de seus clientes e não somente a rentabilidade de seus produtos. Analisar a atual carteira de clientes e quais poderão ser seus clientes em potencial e, focando no atendimento das necessidades dos clientes que oferecem um maior retorno para a empresa, mantendo esses clientes fidelizados, é uma questão que se torna cada vez mais importante.

Em detrimento a isso, a análise da rentabilidade de clientes, que nada mais é do que a apuração dos custos para atender determinado cliente obtendo assim a parcela de margem de 
contribuição para o lucro total da empresa, tem sido considerada de grande importância para o processo de formulação de estratégias e a otimização dos lucros da empresa, pois, através desta análise, indica-se a lucratividade individual de cada cliente.

Conhecer a rentabilidade de cada cliente pode trazer muitos benefícios para a empresa, visto que, dessa forma a empresa pode criar estratégias voltadas a um atendimento mais eficaz dos clientes com maior potencial de rentabilidade, consequentemente melhorando seus resultados. Conforme Bete (2011), alguns dos principais benefícios de se conhecer a rentabilidade dos clientes são:

a) Atender melhor os clientes existentes e que são mais rentáveis. Conhecendo a rentabilidade dos clientes é possível atender aos clientes de forma diferenciada. Pode-se identificar as características dos clientes mais rentáveis e atender essa demanda como: manter um determinado nível de estoque de produtos, atender ao cliente em um prazo menor, dependendo das exigências solicitadas.

b) Conhecer o custo dos serviços prestados e assim poder cobrar pelo serviço disponibilizado. Para os clientes que demandam um grande volume de atividades devese atribuir um maior valor, e não distribuir esse valor com os clientes que não possuem essa demanda elevada.

c) Oferecer descontos aos clientes que são possíveis de atender com custos reduzidos.

d) Atrair potenciais clientes rentáveis. Através de uma análise de volume de compras, localização, estratégia de atuação dos clientes potenciais é possível oferecer descontos e melhorar o nível de serviço atual atraindo esses clientes.

Diante do fato de que deve-se atender melhor os clientes com uma maior margem de lucratividade, Bete (2011) alerta que, também deve-se analisar outras variáveis como, clientes que não são muito rentáveis mas, possuem um grande potencial de compras, clientes novos os quais ainda não é possível definir com precisão sua participação na rentabilidade atual da empresa que, com o tempo podem se tornar mais rentáveis, clientes que valorizam a marca da empresa no mercado, como grandes empresas conhecidas no mercado que agregam valor a marca do fornecedor, tornando-o conhecido por atender esse cliente.

Conforme Farris, et al. (2007), assim como algumas marcas são mais lucrativas do que outras, também o são alguns clientes.

Segundo os mesmos autores a empresa pode ser melhorada tratando clientes distintos de formas diferentes, dessa forma, os clientes podem ser classificados da seguinte maneira:

a) Clientes de primeiro nível - Recompensar: Os clientes de primeiro nível são os mais valiosos que a empresa deve manter, devem receber mais atenção que os outros, pois, perdendo-os os lucros serão afetados. Valorizam o que a empresa faz e podem não ser sensíveis ao preço.

b) Clientes de segundo nível - Desenvolver: São clientes intermediários de lucro baixo a médio que podem ser desenvolvidos e podem se tornar de primeiro nível.

c) Clientes de terceiro nível - Dispensar: A empresa perde dinheiro com esses clientes e não consegue promove-los facilmente, deve ser considerado a possibilidade de cobrar mais pelos serviços.

O entendimento das necessidades dos clientes, considerando os diferentes níveis de lucratividade e ajustando o nível de serviços com base nessas variáveis é fundamental. Através da 
análise da rentabilidade e do entendimento dos custos e receitas que compreendem a equação do lucro, é possível aumentar a lucratividade atual e futura da carteira de clientes. Nesse sentido Zeithaml, Rust e Lemon (2001), propõe um modelo onde os clientes são classificados em função da sua rentabilidade e níveis de atendimento ajustados aos segmentos identificados.

Zeithaml, Rust e Lemon (2001), classificam os níveis que compõem a pirâmide de clientes em:

a) Camada de Platina: Abrange os clientes mais lucrativos da empresa, os grandes usuários do produto, não excessivamente sensíveis ao preço, dispostos a investir e experimentar novas ofertas e comprometidos com a empresa.

b) Camada de Ouro: Difere da de platina nos níveis de lucratividade, que não são tão altos, pelos descontos ou por não serem tão leais. Podem ser grandes usuários que minimizam o risco trabalhando com vários fornecedores.

c) Camada de Ferro: Formada por clientes essenciais, que proveem o volume necessário à utilização da capacidade da empresa, mas, cujos níveis de gastos, lealdade e lucratividade não são suficientes para justificar tratamento especial.

d) Camada Chumbo: Consiste em clientes que oneram a empresa. Exigem mais atenção do que merecem por seus gastos e lucratividade e, às vezes, são problemáticos.

Em relação a isso, segundo Farris, Bendle, Pfeifer e Reibstein (2007), um banco de dados no qual a empresa possa analisar a rentabilidade de clientes individualmente pode ser uma vantagem competitiva, pois, com essas informações, a empresa tem mais chances de defender melhores clientes e até mesmo tomar consumidores rentáveis de seus concorrentes.

Kaplan e Narayana (2001) destacam que o conhecimento da lucratividade das atividades bem como dos pedidos e clientes, permite também identificar situações desfavoráveis à empresa como os produtos oferecido para clientes não lucrativos. Conforme a experiência de Kaplan nesse estudo denominado "Curva das Baleias" observa-se que em geral os $20 \%$ dos clientes mais lucrativos são responsáveis pelo maior lucro acumulado, demonstrando que a menor parte dos clientes são verdadeiramente lucrativos. O gráfico considera a regra 20-80, ou seja, $20 \%$ dos clientes geram $80 \%$ do lucro total da empresa. Porém deve-se observar que essa análise é feita com os dados passados da empresa, portanto deve-se tomar cuidado ao utilizar essas informações na tomada de decisão pois, o cenário pode mudar.

Todas as informações do mercado e suas variáveis devem ser analisadas pela empresa de forma com que seja possível atender de maneira adequada e eficiente todos os segmentos de clientes. Fazer com que a carteira de clientes da empresa seja a mais rentável possível, avaliando também as outras variáveis como, divulgação da marca e volume de produção é um grande desafio que pode ser a chave para o sucesso, pois segundo Padovese (2009), uma rentabilidade adequada continuadamente é, possivelmente, o maior indicador de sobrevivência e sucesso da empresa.

\subsection{Métodos de custeio e mensuração da rentabilidade de clientes}

Os custos de uma empresa são resultados de uma combinação de diversos fatores internos e externos, de modo geral, quanto mais estruturada for a empresa melhores serão os resultados obtidos por meio de um sistema de custos.

A escolha do método de custeio correto para cada perfil de empresa é muito importante, pois, um método inadequado no qual não se avalia os custos de forma correta, distorcendo os 
resultados podendo influenciar negativamente nas decisões estratégicas da empresa. Segundo Megliorini (2011), existem diferentes modelos de custeio, os quais são adotados de acordo com os objetivos estabelecidos pela empresa. Entre os principais métodos utilizados do mercado, podemos destacar:

Custeio por Absorção: Segundo Martins (2010), o custeio por absorção consiste na aplicação de todos os custos de produção aos bens elaborados, e só os de produção são distribuídos para todos os produtos, as despesas administrativas, comerciais e financeiras não integram o custo do produto. Trata-se do único modelo aceito para fins fiscais e legais.

Custeio ABC: Conforme Megliorini (2011), é o método de custeio pelo qual se apropriam os custos dos recursos consumidos em um determinado período às atividades executadas pela empresa, o custo dos produtos resulta, da soma dos custos das atividades necessárias para a sua fabricação. Por esse método de custeio é possível identificar as atividades que agregam valor e as que não agregam valor aos produtos.

Custeio Variável: Segundo Cruz (2011), consiste no método de custeio que separa os custos fixos dos custos variáveis, alocando aos produtos e aos serviços somente os custos variáveis por considerar que os custos fixos são derivados da estrutura da empresa e não dos produtos e serviço. Por esse método custos e despesas também são separados em fixos e variáveis. Como principal funcionalidade, o custeio variável auxilia na identificação da Margem de contribuição.

Conforme Cruz (2011), cada método de custeio apresenta uma série de características e funcionalidades diferentes, havendo a necessidade de o gestor estabelecer o método que melhor atenda seus objetivos. Conforme apresentado no Quadro 1, cada método de custeio possui características diferentes e específicas, cabe aos gestores definirem quais os objetivos que devem ser atingidos, e assim, definir o método de custeio que mais se adapta as atuais necessidades.

Quadro 1- Comparação entre os principais métodos de custeio:

\begin{tabular}{|c|c|}
\hline \multirow{6}{*}{$p^{3}$} & Identifica o consumo dos recursos da empresa (custos) e de gestão da empresa (despesa) \\
\hline & Identifica o custo de cada departamento da organização \\
\hline & $\begin{array}{l}\text { Identificação dos departamentos que tem envolvimento direto com a operação( centro de } \\
\text { custos produtivos) e quais não ( centro de custos auxiliares) }\end{array}$ \\
\hline & Identifica o percentual de custos da empresa que é alocado aos produtos e serviços \\
\hline & Mensuração do custo unitário de cada produto e serviço \\
\hline & Mensura o custo do produto vendido \\
\hline \multirow{6}{*}{ ثิ } & Identifica o consumo dos recursos da empresa (custos) e de gestão da empresa (despesa) \\
\hline & $\begin{array}{l}\text { Identifica o custo de cada processo da empresa } \\
\end{array}$ \\
\hline & Identifica o percentual de custos da empresa que é alocado aos produtos e serviços \\
\hline & Identifica o percentual de custos da empresa que é alocado diretamente aos produtos e serviços \\
\hline & $\begin{array}{l}\text { Identifica as atividades da empresa que tem relação direta com os produtos e serviços e quais } \\
\text { atividades apresentam relação de apoio }\end{array}$ \\
\hline & Mensura o custo do produto vendido \\
\hline \multirow{6}{*}{$3 p^{3}$} & Identifica as receitas separadas por produtos \\
\hline & $\begin{array}{c}\text { Identifica os gastos variáveis por produto, separando as operações dos produtos e serviços da } \\
\text { empresa }\end{array}$ \\
\hline & Identifica a contribuição efetiva de cada produto no pagamento da estrutura da empresa \\
\hline & $\begin{array}{c}\text { Apresenta a margem de contribuição como fator informacional do estabelecimento do preço de } \\
\text { venda }\end{array}$ \\
\hline & Identifica os custos fixos específicos da empresa \\
\hline & $\begin{array}{c}\text { Identifica a composição do lucro da empresa, em suas relações com os produtos e serviços e a } \\
\text { estrutura da empresa }\end{array}$ \\
\hline
\end{tabular}

Fonte: Cruz (2011) 
Para apurar os resultados obtidos com os clientes, torna-se fundamental a escolha de um método de custeio adequado, proporcionando validez a mensuração. Dessa forma, o custeio variável, apontado por Padovese (2009) como uma ferramenta para a determinação do custo dos produtos no processo decisório, passa a ser recomendado também para mensurar a rentabilidade obtida com os clientes. Pois por esse método de custeio, os produtos receberão somente os custos variáveis, os custos fixos são tratados como custo do período indo direto para o resultado do exercício, contribuindo dessa forma para o cálculo da margem de contribuição de cada cliente.

O custeio variável associado a margem de contribuição é considerado uma importante ferramenta para a tomada de decisão pois, através deste, é possível identificar os custos fixos que são da empresa, dos custos variáveis de cada projeto e, através do cálculo da margem de contribuição, é possível verificar quanto cada cliente contribui para cobrir os custos fixos totais e qual sua real rentabilidade.

\subsection{Rentabilidade de clientes através da Margem de Contribuição}

Conforme Farris et al. (2007), a rentabilidade do cliente, é o lucro que a empresa tem com seu atendimento ou de um grupo de clientes no decorrer de um determinado período.

Conforme Guerreiro, Merschmann e Bio (2008), a análise da rentabilidade de um determinado cliente é efetuada a partir da margem de contribuição ou margem bruta dos produtos vendidos a esse cliente, deduzidos os custos para servi-lo.

A margem de contribuição é igual ao valor das vendas de mercadorias e ou serviços prestados, diminuído dos custos e despesas variáveis. Representa a parcela excedente dos custos e das despesas gerados pelo produto, a empresa começa a ter lucro quando a margem de contribuição supera os custos e despesas fixas do período.

Conforme Megliorini (2011), a margem de contribuição é o valor que resta do preço de venda de um produto depois da dedução de seus custos e despesas variáveis. Para Padoveze (2009), o modelo de decisão da margem de contribuição é o modelo decisório fundamental para a gestão dos negócios da empresa, seja em termos de rentabilidade dos produtos, atividades, clientes ou a empresa como um todo. A margem de contribuição de cada produto, linha de produto, clientes etc., é obtida pelo método de custeio variável, o que possibilita aos gestores utiliza-la como ferramenta de auxilio no processo decisório, que conforme Megliorini (2011), inclui ações como:

a) Identificar os produtos, clientes etc. que mais contribuem para a rentabilidade da empresa;

b) Determinar os produtos que podem ter suas vendas incentivadas ou reduzidas e aqueles que podem ser excluídos;

c) Identificar os produtos e serviços e clientes que possuem maior rentabilidade quando existe limitação da produção, permitindo uma melhor tomada de decisão;

d) Definir os preços e condições especiais;

e) Determinar o número mínimo de atividades para que a atividade torne-se rentável;

f) Definir uma melhor negociação com o cliente, e conhecer o limite de desconto permitido.

Para Pereira (2001), a importância da margem de contribuição reside no fato desta ser requerida para a realização da avaliação dos resultados gerados pela empresa. Entretanto, para 
poder obter-se a margem de contribuição, a empresa deve ter os custos apurados de forma correta e segura pois, deles dependem a confiabilidade do valor encontrado.

Dessa maneira, com base nos autores consultados, conclui-se que através da margem de contribuição é possível conhecer a participação de cada produto, serviço ou cliente na formação do resultado da empresa. Se a margem de contribuição não é conhecida, a empresa pode vender muito e estar tendo prejuízo. Alguns clientes podem não contribuir para uma margem aceitável, mas, pode estar relacionada a alguma estratégia promocional de vendas, com o total conhecimento de seus gestores. Portanto, conhecer a margem de contribuição, torna-se fundamental em qualquer processo de determinação da rentabilidade obtida com os clientes.

\section{MATERIAIS E MÉTODOS}

Para atender o objetivo do estudo de identificar e avaliar a rentabilidade dos clientes através da margem de contribuição o presente trabalho foi elaborado sob a ótica de uma pesquisa exploratório-descritiva (SILVA e MENEZES, 2001). Quanto à natureza é aplicada, com característica de um estudo qualitativo (GIL, 1999). Como procedimento técnico foi utilizado o estudo de caso, conforme Gil (1999), o estudo de caso vem sendo utilizado com muita frequência pelos pesquisadores das ciências sociais, pois, possui o propósito de explorar situações da vida real que não estão claramente definidos e descreve a situação do contexto em que está sendo feito o estudo. São utilizados dados concretos permitindo uma melhor avaliação da situação e permite a sugestão de melhorias que podem ser implantadas no processo.

O estudo de caso foi realizado com base nos dados obtidos de uma empresa de médio porte localizada na serra gaúcha fundada em 2010 a partir de uma empresa de móveis sob medida que que recentemente teve a inclusão de três novos sócios buscando um novo mercado de atuação. Atualmente a empresa opera na produção, venda e montagem de móveis para pontos de venda, possuindo significativa participação no mercado onde atua, optou-se por essa empresa para posteriormente poder aplicar de maneira prática o trabalho sugerido.

Optou-se pelo estudo qualitativo de nível exploratório de forma que posteriormente o trabalho possa ser utilizado como base para a gestão de novos negócios da empresa.

Para a realização deste estudo, diferentes técnicas de coleta de dados foram utilizadas, entre elas a análise documental realizada via exame de relatórios contábeis, de custos e de recursos humanos da empresa. De acordo com Sá-Silva, Almeida e Guindani (2009), esse tipo de técnica é caracterizado pela busca de informações em documentos que ainda não receberam nenhum tratamento científico. Também foram analisados os processos de confirmação de pedido, prazo de entrega, fabricação e logística dos clientes, através de um protocolo de estudo onde foram coletados os dados da empresa através de entrevistas não estruturadas com os sócios e os responsáveis por cada setor, apurando os dados necessários para a elaboração desse trabalho, conforme Yin (2010) o protocolo estabelece procedimentos que direcionam a pesquisa.

Após a apuração dos dados, os mesmos foram utilizados para as seguintes mensurações e análises: análise do faturamento e da margem de contribuição de cada cliente, apuração dos custos e classificação dos clientes com base na rua rentabilidade. Após essa análise descritiva, avaliou-se as relações entre as variáveis e posteriormente determinou-se os efeitos resultantes na empresa. Dessa forma, permitindo identificar a rentabilidade dos clientes através da margem de contribuição em uma empresa moveleira de médio porte, proporcionando um melhor gerenciamento de seus recursos e a introdução de uma nova linha de produtos. 


\section{RESULTADOS E DISCUSSÃO}

Nesse tópico são apresentados os resultados por meio do estudo de caso, enfatizando os aspectos da mensuração por meio do custeio variável e das análises de rentabilidade dos clientes.

\subsection{Caracterização da Empresa}

A empresa considerada na análise de caso é uma empresa que opera na produção, venda, distribuição e montagem no segmento de móveis para pontos de venda, com nome fictício de Bella. Recentemente a empresa redesenhou sua área de gerência com a entrada de 3 novos sócios, o que fez com que a empresa adquirisse novos clientes. Tal formato fez com que a empresa crescesse muito em pouco tempo, aumentando o faturamento e consequentemente seus custos operacionais.

A empresa está crescendo e ganhando espaço no mercado, vem cada vez mais sendo procurada por novos clientes, também se tem como objetivo a criação de uma nova linha de produtos, porém, sua estrutura atual não comporta mais a demanda. Diante desse cenário, tonou-se necessária uma apuração mais completa da real rentabilidade de cada cliente, de forma que fosse possível segmentar os clientes por meio de sua rentabilidade, avaliando as reais necessidades de cada um, para dessa forma, gerenciar melhor a carteira dos atuais e potenciais clientes.

Até a implantação da referida segmentação de mercado, a empresa não apurava a rentabilidade de seus clientes para avaliar se o nível de serviço oferecido, estava ou não gerando um relacionamento rentável. A empresa já atua com o custo variável, porém não de uma forma muito definida, sendo assim, verificou-se a necessidade de apurar os custos de forma mais precisa, através do levantamento dos custos fixos da empresa e os custos variáveis para o atendimento de cada cliente. Consequentemente, utilizar esses dados na mensuração da rentabilidade de cada cliente de forma que fosse possível administrar de uma maneira mais lucrativa sua carteira de clientes, introduzindo sua nova linha de produtos na produção.

\subsection{Mensuração dos custos por meio do Custeio Variável.}

Primeiramente foram identificados quais os produtos fabricados pela empresa Bella. Nesse levantamento de dados identificou-se que, a empresa possui vários produtos compondo uma loja completa de mobiliário sendo que cada cliente possui um padrão de loja específico, quiosques de venda de produtos e expositores para pontos de vendas. Como não seria viável o levantamento de cada unidade que compõe uma grande loja, identificou-se uma loja como um produto único para cada cliente. Também se optou por calcular somente os valores dos mobiliários e não da logística, distribuição, entrega e montagem.

A atual carteira da empresa é de 30 clientes, em conversa com a diretoria, sugeriu-se que fosse levantado a rentabilidade de 10 dos principais produtos/clientes e também de seu maior cliente Samsung calculou-se 9 de seus mais relevantes produtos, do seu segundo maior clientes Saraiva estudou-se 2 de seus maiores produtos. Dessa forma, os produtos atuais da empresa são demonstrados na Tabela 01, com os respectivos valores de venda praticados do mês 04/2014. 
Tabela 1- Produtos/Clientes x Preço de Venda

\begin{tabular}{|c|c|c|c|}
\hline CLIENTE & PRODUTO & \multicolumn{2}{|c|}{ RECEITA LÍQUIDA } \\
\hline CLIENTE A - PRODUTO 01 & LOJA DE SÃO PAULO & $\mathrm{R} \$$ & $130.936,18$ \\
\hline CLIENTE A - PRODUTO 02 & QUISQUE 3X4X1,60 & $\mathrm{R} \$$ & $41.172,30$ \\
\hline CLIENTE A - PRODUTO 03 & QUIOSQUE 3X3X1,60 & $\mathrm{R} \$$ & $38.405,75$ \\
\hline CLIENTE A - PRODUTO 04 & QUIOSQUE $3 \times 3 \times 1,40$ & $\mathrm{R} \$$ & $36.484,79$ \\
\hline CLIENTE A - PRODUTO 05 & QUIOSQUE 3X4X1,40 & $\mathrm{R} \$$ & $40.057,61$ \\
\hline CLIENTE A - PRODUTO 06 & QUIOSQUE $3 \times 2 \times 1,60$ & $\mathrm{R} \$$ & $33.037,20$ \\
\hline CLIENTE A - PRODUTO 07 & QUIOSQUE $3 \times 2 \times 1,40$ & $\mathrm{R} \$$ & $32.045,67$ \\
\hline CLIENTE A - PRODUTO 08 & DISCOVERY 1 & $\mathrm{R} \$$ & $5.000,00$ \\
\hline CLIENTE A - PRODUTO 09 & DISCOVERY 2 & $\mathrm{R} \$$ & $5.500,00$ \\
\hline CLIENTE B- PRODUTO 01 & LOJA SOROCABA & $\mathrm{R} \$$ & $171.669,82$ \\
\hline CLIENTE B- PRODUTO 02 & MESA APLLE & $\mathrm{R} \$$ & $3.390,40$ \\
\hline CLIENTE C & QUIOSQUES & $\mathrm{R} \$$ & $29.917,07$ \\
\hline CLIENTE D & LOJA DE CONTAGEM & $\mathrm{RS}$ & $594.339,35$ \\
\hline CLIENTE E & LOJA & $\mathrm{RS}$ & $89.059,11$ \\
\hline CLIENTE F & LOJA & $\mathrm{R} \$$ & $195.200,00$ \\
\hline CLIENTE G & QUIOSQUE & $\mathrm{R} \$$ & $54.982,72$ \\
\hline CLIENTE H & LOJA DE LAJEADO & $\mathrm{R} \$$ & $113.560,97$ \\
\hline CLIENTE I & LOJA DE RIBEIRÃO PRETO & $\mathrm{R} \$$ & $44.073,82$ \\
\hline CLIENTE J & EXPOSITORES & $\mathrm{R} \$$ & $40.000,00$ \\
\hline
\end{tabular}

Fonte: Elaborado pelos autores.

Para esse levantamento de dados analisou-se a representatividade de cada cliente no faturamento total da empresa no ano de 2013. Posteriormente apurou-se os principais produtos vendidos para esses clientes, para que dessa forma a empresa tenha uma visão geral dos principais responsáveis pelo seu faturamento atual e qual a rentabilidade geral de cada um. A Tabela 2 nos mostra o percentual de cada um dos clientes estudados sobre o faturamento da empresa em 2013.

Tabela 2- Percentual sobre o Faturamento de 2013

\begin{tabular}{c|c}
\hline CLIENTE & PERCENTUAL SOBRE O FATURAMENTO \\
\hline CLIENTE A & $15,45 \%$ \\
\hline CLIENTE B & $7,00 \%$ \\
\hline CLIENTE D & $6,43 \%$ \\
\hline CLIENTE J & $4,86 \%$ \\
\hline CLIENTE E & $4,23 \%$ \\
\hline CLIENTE C & $3,89 \%$ \\
\hline CLIENTE I & $3,78 \%$ \\
\hline CLIENTE G & $3,67 \%$ \\
\hline CLIENTE F & $3,50 \%$ \\
\hline CLIENTE H & $3,45 \%$
\end{tabular}

Fonte: Elaborado pelos Autores

Com base nos resultados apurados, conclui-se que os dez clientes estudados foram responsáveis por 56\% do faturamento da empresa em 2013, sendo os outros $44 \%$ divididos entre os outros 20 clientes da empresa, dessa forma estaremos analisando os principais clientes para que os dados apurados sejam relevantes e úteis na tomada de decisão. 
Definidos os clientes/produtos a serem estudados, foram apurados seus custos variáveis. Para apuração desses custos, primeiramente foi feita uma análise nos bancos de dados com o histórico dos custos variáveis, posteriormente foi feita uma reavaliação desses dados através do acompanhamento no setor de compras e da produção de cada tipo de produto de forma que fosse possível identificar da maneira mais correta os custos de cada item.

O primeiro custo variável apurado foi o custo da matéria-prima, para esse cálculo foi identificada a quantidade de matéria-prima consumida na fabricação de cada produto. Posteriormente foram levantados os custos do total de cada matéria-prima, com a multiplicação da quantidade pelo valor, encontrou-se o custo dos materiais diretos empregados a cada produto, os resultados são demonstrados na Tabela 3.

Tabela 3- Custo da Matéria - Prima

\begin{tabular}{|c|c|c|c|c|}
\hline CLIENTE & PRODUTO & \multicolumn{2}{|c|}{ VALOR DA MATÉRIA-PRIMA } & PENCENTUAL SOBRE A RECEITA LÍQUIDA \\
\hline CLIENTE A - PRODUTO 01 & LOJA DESÃO PAULO & $\mathrm{R} \$$ & $24.602,20$ & $18,79 \%$ \\
\hline CLIENTE A - PRODUTO 02 & QUISQUE $3 X 4 X 1,60$ & $\mathrm{R} \$$ & $11.436,75$ & $27,78 \%$ \\
\hline CLIENTE A - PRODUTO 03 & QUIOSQUE 3X3X1,60 & $\mathrm{R} \$$ & $10.106,77$ & $26,32 \%$ \\
\hline CLIENTE A - PRODUTO 04 & QUIOSQUE $3 \times 3 \times 1,40$ & $\mathrm{R} \$$ & $10.424,22$ & $28,57 \%$ \\
\hline CLIENTE A - PRODUTO 05 & QUIOSQUE 3X4X1,40 & $\mathrm{R} \$$ & $10.541,53$ & $26,32 \%$ \\
\hline CLIENTE A - PRODUTO 06 & QUIOSQUE 3X2X1,60 & $\mathrm{R} \$$ & $8.694,00$ & $26,32 \%$ \\
\hline CLIENTE A - PRODUTO 07 & QUIOSQUE 3X2X1,40 & $\mathrm{RS}$ & $9.177,25$ & $28,64 \%$ \\
\hline CLIENTE A - PRODUTO 08 & DISCOVERY 1 & $\mathrm{R} \$$ & $1.315,79$ & $26,32 \%$ \\
\hline CLIENTE A - PRODUTO 09 & DISCOVERY 2 & $\mathrm{R} \$$ & $1.447,36$ & $26,32 \%$ \\
\hline CLIENTE B PRODUTO 01 & LOJA SOROCABA & $\mathrm{R} \$$ & $49.241,37$ & $28,68 \%$ \\
\hline CLIENTE B PRODUTO 02 & MESA APLLE & $\mathrm{R} \$$ & 934,03 & $27,55 \%$ \\
\hline CLIENTE C & QUIOSQUES & $\mathrm{R} \$$ & $11.335,00$ & $37,89 \%$ \\
\hline CLIENTE D & LOJA DE CONTAGEM & $\mathrm{RS}$ & $231.924,25$ & $39,02 \%$ \\
\hline CLIENTE E & LOJA & $\mathrm{R} \$$ & $17.613,45$ & $19,78 \%$ \\
\hline CLIENTE F & LOJA & $\mathrm{R} \$$ & $28.378,92$ & $14,54 \%$ \\
\hline CLIENTE G & QUIOSQUE & $\mathrm{RS}$ & $13.138,09$ & $23,89 \%$ \\
\hline CLIENTE H & LOJA DE LAJEADO & $\mathrm{R} \$$ & $47.083,90$ & $41,46 \%$ \\
\hline CLIENTE I & LOJA DE RIBEIRÃO PRETO & $\mathrm{RS}$ & $13.305,56$ & $30,19 \%$ \\
\hline CLIENTE J & EXPOSITORES & $\mathrm{R} \$$ & $21.996,24$ & $54,99 \%$ \\
\hline
\end{tabular}

Fonte: Elaborado pelos Autores

A Tabela 3 nos mostra o valor despendido em matéria-prima e o percentual sobre a receita líquida, esses valores demonstram que o percentual médio de matéria-prima é de $29,12 \%$ sobre o valor das vendas considerado um bom índice pela diretoria da empresa com base nos valores dos concorrentes, em detrimento a isso destaca-se que, a maior parte dos clientes operam abaixo desse percentual, mas, os clientes $\mathrm{C}, \mathrm{D}, \mathrm{H}, \mathrm{I}$ e $\mathrm{J}$ operam acima do percentual médio com destaque o cliente $\mathrm{J}$ onde esse índice supera os $50 \%$, o que já serve de alerta para uma melhor análise nesse custo.

Apurado o valor da matéria-prima, calculou-se as despesas variáveis, verificou-se que as despesas pagas especificamente pelas vendas realizadas foram identificadas como Impostos sobre às vendas e Comissão de Vendas. Para o cálculo dos impostos foram considerados todos os impostos sobre às vendas sendo: ICMS, IPI, PIS, COFINS, IR E CSSL, como a empresa é tributada pelo Lucro Presumido, creditando-se do ICMS e IPI sobre às compras, apurou-se uma média mensal de impostos de $15,14 \%$ conforme dados apresentados no ano de 2013. A Comissão sobre as vendas, somente é paga sobre alguns projetos no percentual de $7 \%$ conforme Tabela 4. 
Tabela 4- Despesas Variáveis

\begin{tabular}{|c|c|c|c|c|c|}
\hline CLIENTE & PRODUTO & \multicolumn{2}{|c|}{ IMPOSTOS } & \multicolumn{2}{|c|}{ COMISSÕES } \\
\hline CLIENTE A - PRODUTO 01 & LOJA DE SÃO PAULO & $\mathrm{RS}$ & $19.823,74$ & $\mathrm{R} \$$ & $9.165,53$ \\
\hline CLIENTE A - PRODUTO 02 & QUISQUE 3X4X1,60 & $\mathrm{RS}$ & $6.233,49$ & $\mathrm{RS}$ & - \\
\hline CLIENTE A - PRODUTO 03 & QUIOSQUE 3X3X1,60 & $\mathrm{RS}$ & $5.814,63$ & $\mathrm{R} \$$ & - \\
\hline CLIENTE A - PRODUTO 04 & QUIOSQUE $3 \times 3 \times 1,40$ & $\mathrm{RS}$ & $5.523,80$ & $\mathrm{R} \$$ & - \\
\hline CLIENTE A - PRODUTO 05 & QUIOSQUE 3X4X1,40 & $\mathrm{RS}$ & $6.064,72$ & $\mathrm{R} \$$ & - \\
\hline CLIENTE A - PRODUTO 06 & QUIOSQUE 3X2X1,60 & $\mathrm{RS}$ & $5.001,83$ & $\mathrm{RS}$ & - \\
\hline CLIENTE A - PRODUTO 07 & QUIOSQUE 3X2X1,40 & $\mathrm{R} \$$ & $4.851,71$ & $\mathrm{R} \$$ & - \\
\hline CLIENTE A - PRODUTO 08 & DISCOVERY 1 & $\mathrm{R} \$$ & 757,00 & $\mathrm{R} \$$ & - \\
\hline CLIENTE A - PRODUTO 09 & DISCOVERY 2 & $\mathrm{R} \$$ & 832,70 & $\mathrm{R} \$$ & - \\
\hline CLIENTE B PRODUTO 01 & LOJA SOROCABA & $\mathrm{R} \$$ & $25.990,81$ & $\mathrm{R} \$$ & $12.016,89$ \\
\hline CLIENTE B PRODUTO 02 & MESA APLLE & $\mathrm{R} \$$ & 513,31 & $\mathrm{R} \$$ & - \\
\hline CLIENTE C & QUIOSQUES & $\mathrm{R} \$$ & $4.529,44$ & $\mathrm{R} \$$ & - \\
\hline CLIENTE D & LOJA DE CONTAGEM & $\mathrm{R} \$$ & $89.982,98$ & $\mathrm{R} \$$ & - \\
\hline CLIENTE E & LOJA & $\mathrm{R} \$$ & $13.483,55$ & $\mathrm{R} \$$ & - \\
\hline CLIENTE F & LOJA & $\mathrm{RS}$ & $29.553,28$ & $\mathrm{R} \$$ & $6.234,14$ \\
\hline CLIENTE G & QUIOSQUE & $\mathrm{R} \$$ & $8.324,38$ & $\mathrm{R} \$$ & - \\
\hline CLIENTE H & LOJA DE LAJEADO & $\mathrm{R} \$$ & $17.193,13$ & $\mathrm{R} \$$ & $7.949,27$ \\
\hline CLIENTE I & LOJA DE RIBEIRÃO PRETO & $\mathrm{R} \$$ & $6.672,78$ & $\mathrm{R} \$$ & $3.085,17$ \\
\hline CLIENTE J & EXPOSITORES & $\mathrm{R} \$$ & $6.056,00$ & $\mathrm{R} \$$ & - \\
\hline
\end{tabular}

Fonte: Elaborado pelos Autores

$\mathrm{Na}$ Tabela 4 é demonstrado os valores dos impostos sobre as vendas realizadas e o valor das comissões pagas sobre às vendas. $\mathrm{O}$ valor dessas despesas não pode ser reduzido devido ao fato de que tratam-se de percentuais previamente definidos cujo pagamento deve ser efetuado.

Apresentados os valores de venda, os custos e despesas variáveis é possível apurar a margem de contribuição de cada cliente/produto conforme apresentado no próximo capítulo.

\subsection{Cálculo da Margem de Contribuição}

O cálculo da rentabilidade através da Margem de Contribuição dos grupos de clientes/produtos teve como ponte de partida as informações dos bancos de dados da empresa e o faturamento apresentado na Tabela 1. Em seguida foram utilizados os dados derivados da mensuração do custeio e despesas variável apresentados nas Tabelas 3 e 4 .

Os valores da margem de contribuição dos produtos foram determinados com base na fórmula a seguir:

$$
\underline{\mathrm{MCu}}=\mathbf{P V}-\mathrm{CVu}-\mathrm{DVu}
$$

Onde:

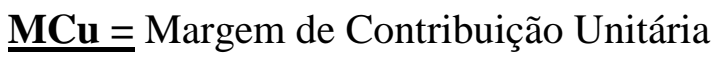

$\underline{\mathbf{P V}}=$ Preço de venda (apurado conforme Tabela 1)

$\underline{\mathbf{C V u}}=$ Custeio Variável Unitário (conforme Tabela 3)

$\underline{\mathbf{D V u}}=$ Despesa Variável Unitária (conforme Tabela 4)

Aplicando-se essa fórmula nos dados coletados, foi apurada a margem de contribuição dos clientes selecionados, conforme demonstrado na Tabela 5. 
Tabela 5- Margem de Contribuição Unitária

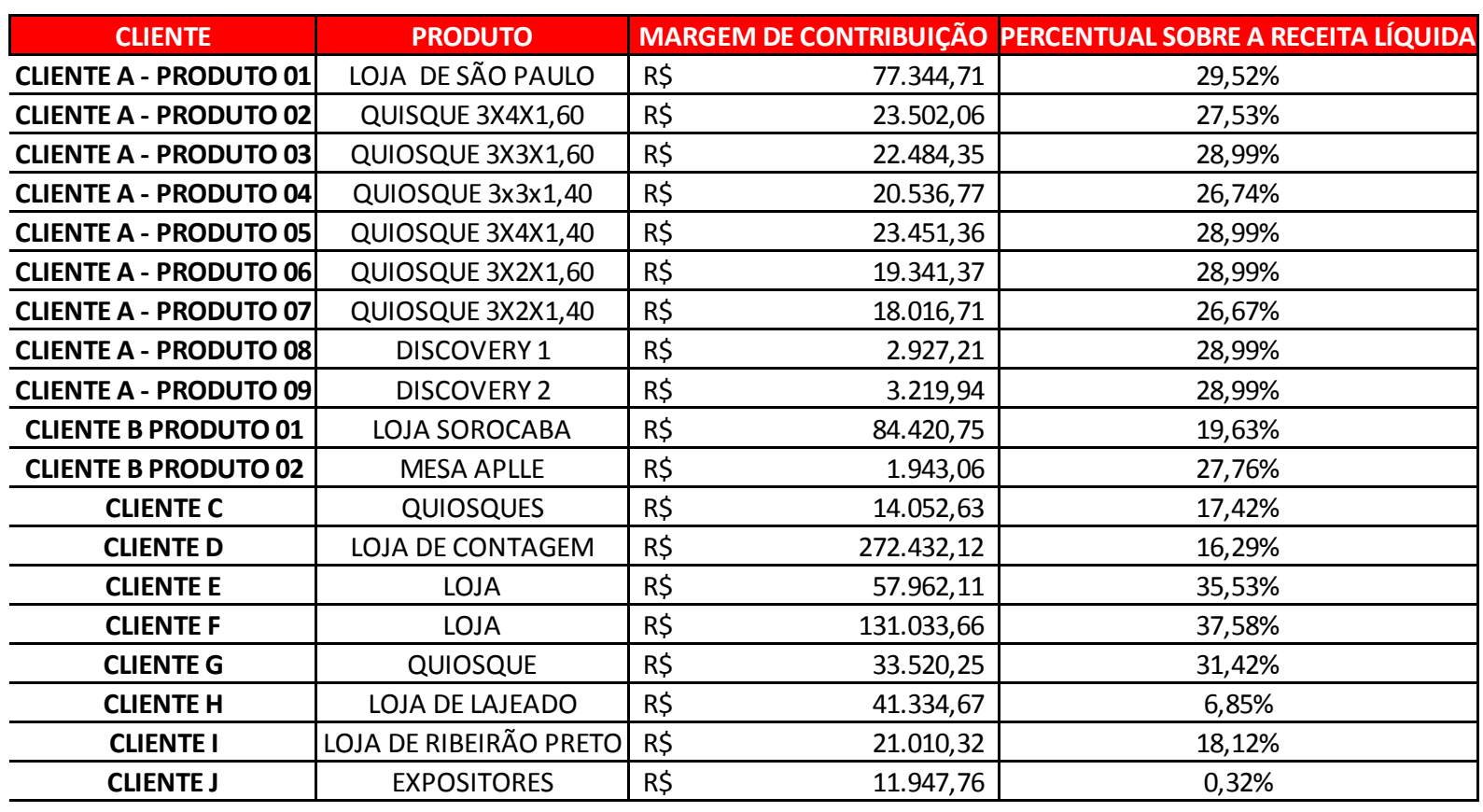

Fonte: Elaborado pelos Autores

Os valores da Margem de Contribuição calculados de cada cliente/produto demonstrado na Tabela 5, evidenciam a parcela que cada um contribui para cobrir os custos fixos da empresa. Os dados apurados demonstram que a média de Margem de Contribuição da empresa é de 54\% e que, a maior parte dos clientes atinge esse patamar. Destacam-se o Cliente $\mathrm{F}$ e E que atingem quase $70 \%$ e também são os clientes que possuem baixo valor de matéria-prima, G, A e B no produto mesas Aplle também superam a média apurada, o restante dos clientes ficam abaixo da média com destaque para o Cliente $\mathrm{J}$ que apresenta somente $29,87 \%$ sendo também o cliente que apresenta o maior valor de matéria-prima consumido.

Em uma análise geral os clientes com maior margem de contribuição estão entre os menos representativos no faturamento geral da empresa e, os maiores responsáveis pelo faturamento Cliente B e Cliente A também superam a média, dessa forma com esses dados apurados é possível trabalhar ajustando a atual carteira de clientes.

\subsection{Lucro Líquido e Ponto de Equilíbrio}

Além de determinar a margem de contribuição, que é muito importante para o gerenciamento do negócio, é fundamental também calcular a rentabilidade que os clientes/produtos proporcionam, sobre o preço de venda.

Conforme Farris, Bendle, Pfeifer e Reibstein (2007), o cálculo da rentabilidade do cliente é um passo importante para entender quais relações com clientes são melhores do que outras e a empresa descobrirá que algumas relações não são lucrativas e por outro lado poderá identificar seus clientes mais rentáveis e tomar iniciativas para garantir a continuação dessas relações. Que nada mais é do que o lucro que a empresa tem com o atendimento de um cliente ou grupo de clientes em um período determinado.

Para melhor analisar a rentabilidade, também foi calculado o Lucro Líquido da empresa em cada projeto, para isso foram utilizados os dados de receita de vendas apresentados anteriormente 
na Tabela 01, reduzindo os custos e despesas variáveis apurados na Tabela 03, 04, apresentando a margem de contribuição Tabela 05.

Existe uma dificuldade em apurar de maneira precisa os custos fixos da empresa pois, através do custeio variável, os custos e despesas fixas são tratados como custo do produto e vão direto para o resultado. Para que fosse possível apurar o Lucro Líquido, utilizou-se um critério de rateio onde, o total de custos fixos da empresa que atualmente é de $\mathrm{R} \$ 448.500,66$, foi dividido entre os clientes/produtos com base no percentual sobre a receita líquida conforme tabela 6.

Tabela 6: Distribuição do percentual de Custo Fixo sobre a Receita Líquida

\begin{tabular}{|c|c|c|c|c|}
\hline CLIENTE & PRODUTO & \multicolumn{2}{|c|}{ RECEITA LÍQUIDA } & PERCENTUAL SOBRE A RECEITA LÍQUIDA TOTAL \\
\hline CLIENTE A - PRODUTO 01 & LOJA DE SÃO PAULO & $\mathrm{R} \$$ & $130.936,18$ & $7,71 \%$ \\
\hline CLIENTE A - PRODUTO 02 & QUISQUE 3X4X1,60 & $\mathrm{R} \$$ & $41.172,30$ & $2,42 \%$ \\
\hline CLIENTE A - PRODUTO 03 & QUIOSQUE 3X3X1,60 & $\mathrm{R} \$$ & $38.405,75$ & $2,26 \%$ \\
\hline CLIENTE A - PRODUTO 04 & QUIOSQUE $3 \times 3 \times 1,40$ & $\mathrm{RS}$ & $36.484,79$ & $2,15 \%$ \\
\hline CLIENTE A - PRODUTO 05 & QUIOSQUE 3X4X1,40 & $\mathrm{RS}$ & $40.057,61$ & $2,36 \%$ \\
\hline CLIENTE A - PRODUTO 06 & QUIOSQUE 3X2X1,60 & $\mathrm{R} \$$ & $33.037,20$ & $1,94 \%$ \\
\hline CLIENTE A - PRODUTO 07 & QUIOSQUE 3X2X1,40 & $\mathrm{R} \$$ & $32.045,67$ & $1,89 \%$ \\
\hline CLIENTE A - PRODUTO 08 & DISCOVERY 1 & $\mathrm{R} \$$ & $5.000,00$ & $0,29 \%$ \\
\hline CLIENTE A - PRODUTO 09 & DISCOVERY 2 & $\mathrm{RS}$ & $5.500,00$ & $0,32 \%$ \\
\hline CLIENTE B PRODUTO 01 & LOJA SOROCABA & $\mathrm{R} \$$ & $171.669,82$ & $10,11 \%$ \\
\hline CLIENTE B PRODUTO 02 & MESA APLLE & $\mathrm{R} \$$ & $3.390,40$ & $0,20 \%$ \\
\hline CLIENTE C & QUIOSQUES & $\mathrm{RS}$ & $29.917,07$ & $1,76 \%$ \\
\hline CLIENTE D & LOJA DE CONTAGEM & $\mathrm{R} \$$ & $594.339,35$ & $34,99 \%$ \\
\hline CLIENTE E & LOJA & $\mathrm{R} \$$ & $89.059,11$ & $5,24 \%$ \\
\hline CLIENTE F & LOJA & $\mathrm{R} \$$ & $195.200,00$ & $11,49 \%$ \\
\hline CLIENTE G & QUIOSQUE & $\mathrm{R} \$$ & $54.982,72$ & $3,24 \%$ \\
\hline CLIENTE H & LOJA DE LAJEADO & $\mathrm{RST}$ & $113.560,97$ & $6,68 \%$ \\
\hline CLIENTE I & LOJA DE RIBEIRÃO PRETO & $\mathrm{R} \$$ & $44.073,82$ & $2,59 \%$ \\
\hline \multirow[t]{2}{*}{ CLIENTE J } & EXPOSITORES & $\mathrm{R} \$$ & $40.000,00$ & $2,35 \%$ \\
\hline & TOTAL & $\mathbf{R} \mathbf{S}$ & $1.698 .832,76$ & $100,00 \%$ \\
\hline
\end{tabular}

Fonte: Elaborado pelos Autores

Aplicado o percentual de custo fixo rateado para cada cliente/produto sobre a Receita Líquida obteve-se o valor dos custos fixos que serão absorvidos por cada cliente/produto, destaca-se que essa forma não é a mais correta, visto que, não apura o real valor de custo fixo para cada cliente, para que fosse possível uma apuração mais eficiente seria necessário o cálculo do custeio por meio do $\mathrm{ABC}$, que não é o objetivo do estudo.

Diminuindo da Margem de Contribuição, os Custos Fixos apurados, encontramos o Lucro Líquido de cada cliente conforme Tabela 7.

Atribuindo a cada produto o valor de custos fixo definido, encontramos o lucro líquido de cada cliente/produto, o percentual desejado pelos diretores da empresa é de 30\%, a média encontra é de $27,69 \%$, bem próximo ao desejado. Cinco clientes superaram o percentual desejado, o Cliente A com maior representatividade no faturamento, atinge 31,53\% de lucro líquido o que é muito bom para a empresa, o segundo maior Cliente B atinge o percentual desejado somente em um de seus produtos, ficando as lojas abaixo do desejado. Os clientes E, F e G são os que apresentam um maior Lucro Líquido, porém possuem pouca representatividade. O restante dos clientes fica abaixo do índice considerado ideal, porém, nenhum deles apresenta percentual zero ou negativo que é muito bom. 
Tabela 7: Lucro Líquido

\begin{tabular}{|c|c|c|c|c|c|c|c|}
\hline CLIENTE & PRODUTO & \multicolumn{2}{|c|}{ MARGEM DE CONTRIBUIÇÃO } & (-) CUSTO FIXO & \multicolumn{2}{|c|}{ LUCRO LÍQUIDO } & \multirow{2}{*}{\begin{tabular}{|c|} 
PERCENTUAL SOBRE A RECEITA LÍQUIDA \\
$32,67 \%$
\end{tabular}} \\
\hline CLIENTE A - PRODUTO 01 & LOJA DE SÃO PAULO & $\mathrm{R} \$$ & $77.344,71$ & RS $34.567,83$ & $\mathrm{R} \$$ & $42.776,88$ & \\
\hline CLIENTE A - PRODUTO 02 & QUISQUE 3X4X1,60 & $\mathrm{R} \$$ & $23.502,06$ & $\mathrm{R} \$ \quad 10.869,70$ & $\mathrm{R} \$$ & $12.632,36$ & $30,68 \%$ \\
\hline CLIENTE A - PRODUTO 03 & QUIOSQUE 3X3X1,60 & $\mathrm{R} \$$ & $22.484,35$ & $\mathrm{R} \$ 10.139,32$ & $\mathrm{R} \$$ & $12.345,03$ & $32,14 \%$ \\
\hline CLIENTE A - PRODUTO 04 & QUIOSQUE $3 \times 3 \times 1,40$ & $\mathrm{R} \$$ & $20.536,77$ & $\mathrm{R} \$ \quad 9.632,17$ & $\mathrm{R} \$$ & $10.904,60$ & $29,89 \%$ \\
\hline CLIENTE A - PRODUTO 05 & QUIOSQUE 3X4X1,40 & $\mathrm{RS}$ & $23.451,36$ & $\mathrm{R} \$ \quad 10.575,42$ & $\mathrm{R} \$$ & $12.875,94$ & $32,14 \%$ \\
\hline CLIENTE A - PRODUTO 06 & QUIOSQUE 3X2X1,60 & $\mathrm{RS}$ & $19.341,37$ & $8.721,99$ & $\mathrm{R} \$$ & $10.619,38$ & $32,14 \%$ \\
\hline CLIENTE A - PRODUTO 07 & QUIOSQUE $3 \times 2 \times 1,40$ & $\mathrm{RS}$ & $18.016,71$ & $8.460,22$ & $\mathrm{R} \$$ & $9.556,48$ & $29,82 \%$ \\
\hline CLIENTE A - PRODUTO 08 & DISCOVERY 1 & $\mathrm{RS}$ & $2.927,21$ & $1.320,03$ & $\mathrm{R} \$$ & $1.607,18$ & $32,14 \%$ \\
\hline CLIENTE A - PRODUTO 09 & DISCOVERY 2 & $\mathrm{R} \$$ & $3.219,94$ & $\mathrm{R} \$ \quad 1.452,03$ & $\mathrm{R} \$$ & $1.767,91$ & $32,14 \%$ \\
\hline CLIENTE B PRODUTO 01 & LOJA SOROCABA & $\mathrm{R} \$$ & $84.420,75$ & $\mathrm{R} \$ \quad 45.321,72$ & $\mathrm{R} \$$ & $39.099,03$ & $22,78 \%$ \\
\hline CLIENTE B PRODUTO 02 & MESA APLLE & $\mathrm{R} \$$ & $1.943,06$ & $\mathrm{R} \$ \quad 895,08$ & $\mathrm{R} \$$ & $1.047,98$ & $30,91 \%$ \\
\hline CLIENTE C & QUIOSQUES & $\mathrm{RS}$ & $14.052,63$ & $7.898,26$ & $\mathrm{R} \$$ & $6.154,36$ & $20,57 \%$ \\
\hline CLIENTE D & LOJA DE CONTAGEM & $\mathrm{R} \$$ & $272.432,12$ & $\mathrm{R} \$ 156.908,67$ & $\mathrm{R} \$$ & $115.523,45$ & $19,44 \%$ \\
\hline CLIENTE E & LOJA & $\mathrm{R} \$$ & $57.962,11$ & $\mathrm{R} \$ \quad 23.512,07$ & $\mathrm{R} \$$ & $34.450,04$ & $38,68 \%$ \\
\hline CLIENTE F & LOJA & $\mathrm{R} \$$ & $131.033,66$ & $\mathrm{R} \$ \quad 51.533,81$ & $\mathrm{R} \$$ & $79.499,85$ & $40,73 \%$ \\
\hline CLIENTE G & QUIOSQUE & $\mathrm{R} \$$ & $33.520,25$ & $\mathrm{R} \$ \quad 14.515,72$ & $\mathrm{R} \$$ & $19.004,52$ & $34,56 \%$ \\
\hline CLIENTE H & LOJA DE LAJEADO & $\mathrm{R} \$$ & $41.334,67$ & $\mathrm{R} \$ 29.980,69$ & $\mathrm{R} \$$ & $11.353,99$ & $10,00 \%$ \\
\hline CLIENTE I & LOJA DE RIBEIRÃO PRETO & $\mathrm{RS}$ & $21.010,32$ & $\mathrm{R} \$ \mathbf{1 1 . 6 3 5 , 7 2}$ & $\mathrm{R} \$$ & $9.374,60$ & $21,27 \%$ \\
\hline CLIENTE J & EXPOSITORES & $\mathrm{R} \$$ & $11.947,76$ & $\mathrm{R} \$ \mathbf{1 0 . 5 6 0 , 2 1}$ & $\mathrm{RS}$ & $1.387,55$ & $3,47 \%$ \\
\hline
\end{tabular}

Fonte: Elaborado pelos Autores

Percebe-se que os clientes que possuem uma maior margem de contribuição são os responsáveis também pelo maior percentual de lucro líquido.

Calculado o Lucro Líquido de cada cliente/produto, apurou-se o ponto de equilíbrio de cada um. O ponto de equilíbrio informa o faturamento mensal mínimo necessário para cobrir os custos, informação que é muito importante para análise da viabilidade do negócio ou a adequação da empresa com o mercado. Para o cálculo do Ponto de Equilíbrio em R \$ de cada cliente, dividiu-se os custos fixos informados na Tabela 7 pelo índice da Margem de contribuição informado na Tabela 5. Os dados obtidos são apresentados na Tabela 8. Esses dados nos mostram o quanto em valor a empresa deve vender para cada cliente/produto para cobrir seus custos e despesas, ou seja, para ter lucro zero, a partir desse valor a empresa começa a ter lucro. Observa-se que os clientes que possuem menos lucro líquido são os que possuem o maior valor em ponto de equilíbrio, ou seja, mais produtos devem ser vendidos para que se tenha lucro em comparação aos produtos que apresentam um menor ponto de equilíbrio em relação a receita líquida.

Tabela 8: Ponto de Equilíbrio

\begin{tabular}{|c|c|c|c|c|c|}
\hline CLIENTE & PRODUTO & \multicolumn{2}{|c|}{ RECEITA LÍQUIDA } & \multicolumn{2}{|c|}{ PONTO DE EQUILIBRIO } \\
\hline CLIENTE A - PRODUTO 01 & LOJA DE SÃO PAULO & $\mathrm{R} \$$ & $130.936,18$ & $\mathrm{R} \$$ & $58.519,58$ \\
\hline CLIENTE A - PRODUTO 02 & QUISQUE 3X4X1,60 & $\mathrm{RS}$ & $41.172,30$ & $\mathrm{R} \$$ & $19.042,18$ \\
\hline CLIENTE A - PRODUTO 03 & QUIOSQUE 3X3X1,60 & $\mathrm{RS}$ & $38.405,75$ & $\mathrm{RS}$ & $17.319,07$ \\
\hline CLIENTE A - PRODUTO 04 & QUIOSQUE $3 \times 3 \times 1,40$ & $\mathrm{RS}$ & $36.484,79$ & $\mathrm{RS}$ & $17.112,13$ \\
\hline CLIENTE A - PRODUTO 05 & QUIOSQUE 3X4X1,40 & $\mathrm{R} \$$ & $40.057,61$ & $\mathrm{RS}$ & $18.064,03$ \\
\hline CLIENTE A - PRODUTO 06 & QUIOSQUE 3X2X1,60 & $\mathrm{RS}$ & $33.037,20$ & $\mathrm{R} \$$ & $14.898,13$ \\
\hline CLIENTE A - PRODUTO 07 & QUIOSQUE 3X2X1,40 & $\mathrm{R} \$$ & $32.045,67$ & $\mathrm{RS}$ & $15.047,90$ \\
\hline CLIENTE A - PRODUTO 08 & DISCOVERY 1 & $\mathrm{RS}$ & $5.000,00$ & $\mathrm{R} \$$ & $2.254,75$ \\
\hline CLIENTE A - PRODUTO 09 & DISCOVERY 2 & $\mathrm{R} \$$ & $5.500,00$ & $\mathrm{RS}$ & $2.480,22$ \\
\hline CLIENTE B PRODUTO 01 & LOJA SOROCABA & $\mathrm{RS}$ & $171.669,82$ & $\mathrm{R} \$$ & $92.161,84$ \\
\hline CLIENTE B PRODUTO 02 & MESA APLLE & $\mathrm{RS}$ & $3.390,40$ & $\mathrm{RS}$ & $1.561,81$ \\
\hline CLIENTE C & QUIOSQUES & $\mathrm{RS}$ & $29.917,07$ & $\mathrm{RS}$ & $16.814,85$ \\
\hline CLIENTE D & LOJA DE CONTAGEM & $\mathrm{RS}$ & $594.339,35$ & $\mathrm{RS}$ & $342.312,78$ \\
\hline CLIENTE E & LOJA & $\mathrm{RS}$ & $89.059,11$ & $\mathrm{RS}$ & $36.126,42$ \\
\hline CLIENTE F & LOJA & $\mathrm{RS}$ & $195.200,00$ & $\mathrm{RS}$ & $76.769,59$ \\
\hline CLIENTE G & QUIOSQUE & $\mathrm{RS}$ & $54.982,72$ & $\mathrm{RS}$ & $23.809,91$ \\
\hline CLIENTE H & LOJA DE LAJEADO & $\mathrm{R} \$$ & $113.560,97$ & $\mathrm{RS}$ & $82.367,55$ \\
\hline CLIENTE I & LOJA DE RIBEIRÃO PRETO & $\mathrm{RS}$ & $44.073,82$ & $\mathrm{RS}$ & $24.408,51$ \\
\hline CLIENTE J & EXPOSITORES & $\mathrm{R} \$$ & $40.000,00$ & $\mathrm{RS}$ & $35.354,60$ \\
\hline
\end{tabular}

Fonte: Elaborado pelos Autores 


\subsection{Análise da Rentabilidade dos Clientes}

Apurados todos os dados necessários para uma análise na carteira atual de clientes, elaborou-se a Pirâmide de clientes conforme a classificação de Zeithaml, Rust e Lemon (2001) com base na margem de contribuição de cada cliente/produto.

Optou-se por fazer essa análise com base na margem de contribuição pois, o rateio das despesas fixas foi feito com base no percentual de cada cliente sobre o faturamento, o que pode gerar distorções nessa análise.

Para elaboração da pirâmide, foi analisado junto aos diretores da empresa qual o percentual ideal de margem de contribuição, definindo-se que acima de $60 \%$ é considerado um percentual ótimo, até $50 \%$ seria considerado bom, até $40 \%$ seria regular e abaixo de $40 \%$, péssimo. A ideia principal dessa pirâmide é que a empresa possa utiliza-la no conhecimento das diferentes rentabilidades de seus clientes para administrar a manutenção ou aumento dessa lucratividade.

No caso da empresa, encontra-se na camada de platina os clientes mais rentáveis ou percentual ótimo, são 3 clientes que não representam muito no faturamento total da empresa, dessa forma deve-se trabalhar mais com esses clientes procurando quais são as suas necessidades para que aumente sua representação.

A maior parte dos clientes/ produtos encontram-se na camada de Ouro ou percentual bom onde, os níveis de lucratividade, não são tão altos, e são grandes usuários que minimizam o risco trabalhando com vários fornecedores o que justamente acontece com esses clientes, possuem mais de um fornecedor para minimizarem o seu risco, nessa camada encontram-se os maiores responsáveis pelo faturamento da empresa, devido a isso, também deve-se atender esses clientes de uma forma especial pois, mesmo que não sejam os mais rentáveis são os maiores responsáveis pelo faturamento atual da empresa.

Quatro clientes/produtos encontram-se na camada de ferro ou percentual regular que é formada por clientes essenciais, que proveem o volume necessário à utilização da capacidade da empresa, mas, cujos níveis de gastos, lealdade e lucratividade não são suficientes para justificar tratamento especial, porém conforme a diretoria da empresa, esses clientes ainda devem ser mantidos devido ao reconhecimento que geram para a empresa em trabalhar com essas marcas.

Dois clientes encontram-se na camada de Chumbo ou percentual péssimo onde os clientes que geram muito pouca lucratividade, definiu-se que esses clientes serão então extinguidos, visto que atualmente não agregam nenhum valor para a empresa e sua margem de contribuição está muito aquém da desejada, e dessa forma será possível inserir a linha de produtos próprios da empresa.

Ao final do estudo verifica-se que, analisar a rentabilidade dos clientes é muito importante para a gestão do negócio, com essas informações, a empresa pode tomar decisões para melhoria de sua lucratividade e também buscar novas alternativas como é o caso da empresa em estudo que quer adotar uma linha própria de produtos. Para isso, conhecer a real situação de seus clientes é fundamental para uma análise geral de sua carteira e com essas informações decidir quais dos clientes devem ser mantidos, avaliados, tentar uma nova negociação e em último caso extinguir para que novas oportunidades possam ser beneficiadas. 


\section{CONSIDERAÇÕES FINAIS}

Cada empresa desenvolve sua análise da rentabilidade de clientes, o melhor método é o que permite aperfeiçoar sua rentabilidade e consequentemente sua carteira de clientes, o sucesso dessa análise depende da correta apuração de dados na empresa.

$\mathrm{Na}$ análise da rentabilidade de clientes da empresa Bella, encontraram-se dificuldades na obtenção de dados na empresa, pois a mesma não tinha um sistema de custeio bem definido o que fez com que foi necessário o acompanhamento de todo processo de compras e faturamento de cada cliente estudado. Existe também uma certa limitação da análise do Lucro Líquido da empresa pois, utilizou-se o método de custeio por absorção rateando os custos fixos da empresa, o que pode prejudicar uma análise correta da rentabilidade pois, os clientes podem ser responsáveis por uma carga de custos fixos que não corresponde à realidade. Dessa forma para que fosse possível uma melhor análise dos resultados, utilizou-se como base sua margem de contribuição, conforme definido no início do estudo.

Para que fosse possível obter os resultados de forma mais precisa, seria necessário apurar os custos de cada cliente conforme o sistema $\mathrm{ABC}$, porém devido à dificuldade de obtenção de dados não foi possível, dessa forma sugere-se que a empresa futuramente adote esse sistema de custeio para obter resultados ainda melhores. Apesar das dificuldades encontradas, a análise da rentabilidade dos clientes através da margem de contribuição foi concluída com sucesso e as vantagens obtidas pela empresa foram muitas, dentre elas, conhecer seus clientes mais rentáveis sendo possível dar mais atenção a eles, conhecer seus clientes menos rentáveis mas que divulgam a imagem da empresa, conhecer seus clientes menos rentáveis decidindo extingui-los para poder fabricar sua própria marca de produtos que é um dos objetivo da empresa para o próximo ano.

Conhecer a rentabilidade dos clientes da empresa pode minimizar suas dificuldades, trata-se de um trabalho significativo que consome tempo. Como qualquer outra atividade de planejamento e controle, apesar das dificuldades encontradas, muitas foram as melhorias obtidas pela empresa o que fez com que a empresa adotasse esse projeto, sendo dessa forma possível afirmar que conhecer a rentabilidade de seus clientes proporcionou resultados eficientes, fazendo com que o objetivo deste trabalho fosse alcançado.

Dessa forma concluo afirmando que a empresa Bella está no caminho certo para tornar a análise da rentabilidade de clientes uma ferramenta de gestão para a obtenção de melhores resultados. Porém melhorias sempre devem ser feitas em todos os processos, dessa forma após dado o primeiro passo, sugere-se que sejam feitas melhorias, principalmente no setor de custos da empresa que ainda é feito de forma muito superficial sem a obtenção concreta de dados, para que dessa forma, a empresa possa melhorar ainda mais sua gestão aperfeiçoando-se cada vez mais, contribuindo assim para melhores resultados a cada ano.

\section{REFERÊNCIAS}

BETE, A. Análise da Rentabilidade de Clientes. Site: Amigonerd.cm. (2011)

CRUZ, J. A. W. Gestão de custos: Perspectivas e Funcionalidades. Curitiba, Ibpex, 2011.

FARRIS, P. W.; BENDLE, Neil T.; PFEIFER P. E.; REIBSTEIN, D. J. Métricas de Marketing: Mais de 50 métricas que todo executivo deve dominar. Porto Alegre, Bookman, 2007.

GIL, A. C. Métodos e técnicas de Pesquisa: Pesquisa social. São Paulo, Atlas, 1999.

GUERREIRO. R. A Meta da Empresa: Seu alcance sem mistérios. 2ed. São Paulo: Atlas, 1999. 
GUERREIRO, R.; BIO, S. R.; MERSCHMANN, E. Cost-to-serve measurement and costumer profitability analysis. International Journal of Logistics. Management, 2008.

KAPLAN, R.S; NARAYANA, V.G. Measuring and managing customer profitability. Cost Management Boston, 2001.

MALHOTRA, N. K. Pesquisa de marketing: foco na decisão. 3. ed. São Paulo: Pearson, 2010.

MARTINS, E. Contabilidade de custos. 10. ed. São Paulo: Atlas, 2010.

MEGLiORINI, E. Custos Análise e Gestão. $3^{\circ}$ Ed. São Paulo, Pearson Prentice Hall, 2011.

OLIVEIRA, S. L. Tratado de metodologia científica. 2. Ed. São Paulo: Pioneira,1999

PADOVEZE, C. L. Controladoria estratégica e operacional: conceitos, estrutura, aplicações.2 ed.rev. e atual. São Paulo: Cengage Learning, 2009.

PEREIRA, C.A. Avaliação de Resultados e Desempenhos. In: CATELLI, A. (Org). Controladoria: uma abordagem da gestão econômica. 2 ed. São Paulo: Atlas, 2001.

SÁ-SILVA, J.R.; ALMEIDA, C.D.; GUINDANI, J.F. Pesquisa documental: pistas teóricas e metodológicas. Revista Brasileira de História \& Ciências Sociais, São Leopoldo, ano 1, n.1, p. 1-15, jul. 2009.

SHARMANN, P. ABC And the botton line on customers. CMA Magazine, p.20-26, Sept.1996.

SILVA, E.L; MENEZES, E.M. Metodologia da pesquisa e elaboração da dissertação. 3 Ed. Florianópolis: Laboratório de Ensino a Distância da UFSC, 2001.

YIN, R.K. Estudo De caso: planejamento e métodos. Porto Alegre, Bookman, 2010.

ZEITHAML, V.A; RUST, R.T; LEMON, K.N. The customer pyramid: creating and serving profitable customers. California Menagement Review, Berkeley, 2001.

Versão preliminar deste artigo foi apresentada na Mostra de pós-graduação da UCS 